\title{
Breaking face processing tasks apart to improve their predictive value in the real world: A comment on Ramon, Bobak, and White (2019)
}

\author{
Christel Devue* (D)
}

School of Psychology, Victoria University of Wellington, New Zealand

\begin{abstract}
In this commentary, I will expand on three aspects suggested by Ramon et al. (2019, British Journal of Psychology, ||$|0,46|$ ) to improve the predictive value of laboratory-based tasks in real-world applications. There are potential benefits that may arise from three interrelated considerations, particularly in terms of predicting agents' susceptibility to errors in operational settings. The first is a proposal to conduct a detailed examination of performance on face processing tests rather than only analysing overall accuracy scores. The second involves considering non-face-related cognitive and meta-cognitive subprocesses involved in face processing tasks. The third highlights the contribution of superior recognisers in creating challenging tests that simulate difficult real-world situations.
\end{abstract}

In the context of a growing demand for people with superior face processing skills to fulfil security-related jobs, Ramon, Bobak, and White (2019) point out that empirical evidence to justify this enthusiasm is currently lacking. Crucially, we know too little about the qualities that make someone superior at face processing, and the correspondence between laboratory-based tasks that are used for selection of 'super-recognisers' and realworld tasks is poor. The authors propose a bi-directional collaboration between face recognition researchers and facial identification practitioners to develop a better understanding of superior face recognition abilities, and whether and how these skills can best be exploited in the field. The suggested approach, where expertise knowledge in both areas would enrich each other, seems particularly relevant and promising.

To improve the predictive value of laboratory-based tests, three points the authors make seem particularly interesting to expand on. They concern (1) the links between laboratory-based tests and real-world tasks, (2) face-related sub-processes involved in real-world tasks, and (3) collaboration between researchers, super-recognisers, and practitioners.

First, the authors highlight a need to increase the correspondence between performance on laboratory-based tests and performance on tasks agents execute in field work, which seems an essential step. A complementary way to increase this correspondence could be via the examination of the specifics of performance rather than overall scores. Superior recognisers are not necessarily superior in all face processing tasks, or in all aspects of a given task (Bate et al., 2019; Bobak, Bennetts, Parris, Jansari, \& Bate, 2016;

\footnotetext{
*Correspondence should be addressed to Christel Devue, School of Psychology, Victoria University of Wellington, PO Box 600, Wellington 6040, New Zealand (email: christel.devue@vuw.ac.nz).
} 
Devue, Wride, \& Grimshaw, 2019). Two examples: (1) superior recognisers perform well on a challenging recognition task not because they remember more faces, but because they make less false alarms than other people (Devue et al., 2019) and (2) the pattern of individual performance of police officers with superior skills varies on match and mismatch trials of a matching task (Bate et al., 2019). It is not uncommon for performance on match and mismatch trials to be uncorrelated (Megreya \& Burton, 2007), but there can also be a negative association between the two, where good performance on one type of trial is associated with worse performance on the other (Reedy \& Devue, 2019). In practice, this could mean that someone good at recognising matching pairs of images may also be more susceptible to accept mismatching pairs of identities. Therefore, sensitivity measures may be better suited than overall accuracy scores for estimating superior performance (Bate et al., 2019), and detailed examinations of performance would provide better estimates of the type of errors agents are susceptible to commit. Since different errors (e.g. misses vs. false alarms) may not create the same risk at border control, in crowd surveillance, in visa or passport applications, or in a CCTV image processing room, it seems necessary to consider performance indices separately, and balance their interpretation against the costs that specific errors incur in the context of a given realworld procedure.

Second, the authors pertinently argue that it is essential to determine the specific facerelated sub-process or combination of sub-processes involved in laboratory-based tasks (i.e. detection, discrimination, recognition, identification) and whether they match subprocesses involved in real-world tasks. It is also crucial to consider more general cognitive processes and meta-cognitive aspects, and how they may play out given the specific constraints and contingencies of a real-world procedure. For example, a laboratory-based matching task typically consists of 50\% match trials and 50\% mismatch trials. Decision processes arising in that situation may differ from those in operational settings that are perhaps more prone to biased decisions (e.g. Larkin, 1971). Indeed, in practice border officers may be rarely confronted with cases of mismatching identities, while immigration officers verifying fraudulent duplicate visa applications would more often deal with cases of mismatching identities. Moreover, the level of insight people have into their own performance may also be important to evaluate in conjunction with objective task performance. In the general population, the correspondence between performance and self-reported abilities is undesirably low (Palermo et al., 2017), and confidence ratings are non-predictive of errors on individual trials (Devue et al., 2019). Therefore, it seems crucial to implement ways to select people who are able to realize when their judgements are uncertain and to proactively consult their peers to reach more accurate decisions.

Finally, the authors highlight potential benefits of combining the expertise of practitioners and the skills of super-recognisers to improve the quality of evidence in forensic settings. Another benefit of such a combination of skills, added to the methodological skills of scientists, would be in creating highly challenging selection tasks that are not only relevant to the real world, but also difficult enough to elicit errors. Since the best recognisers perform at, or close to, ceiling on many existing tasks, their limits are difficult to establish and so these tasks are uninformative of the type of errors they may commit in the field. Therefore, superior recognisers, especially those who are also experienced at a particular field role, may be particularly helpful in assisting scientists to create highly challenging simulations of real-world duties. For example, the difficulty of a matching task can be increased by selecting very similar mismatching pairs of photos or dissimilar pairs of matching identities (Kemp, Caon, Howard, \& Brooks, 2016), and superrecognisers may be best equipped to help scientists with the selection of such test images. 
In conclusion, there will be vast mutual benefits for face recognition researchers and practitioners in the approaches proposed by Ramon et al. (2019), and the years ahead are really exciting. I share the authors' hope that our understanding of human face processing will progress immensely from this endeavour, and that it will create a fairer society.

\section{Acknowledgements}

Warm thanks to Hazel Godfrey for her comments on the draft of this paper.

\section{References}

Bate, S., Frowd, C. D., Bennetts, R. J., Hasshim, N., Portch, E., Murray, E., \& Dudfield, G. (2019). The consistency of super face recognition skills in police officers. Applied Cognitive Psychology, $1-15$.

Bobak, A. K., Bennetts, R. J., Parris, B. A., Jansari, A., \& Bate, S. (2016). An in-depth cognitive examination of individuals with superior face recognition skills. Cortex, 82, 48-62. https://doi. org/10.1016/j.cortex.2016.05.003

Devue, C., Wride, A., \& Grimshaw, G. M. (2019). New insights on real-world human face recognition. Journal of Experimental Psychology: General, 148, 994-1007. https://doi.org/10.1037/xge 0000493

Kemp, R. I., Caon, A., Howard, M., \& Brooks, K. R. (2016). Improving unfamiliar face matching by masking the external facial features. Applied Cognitive Psychology, 30, 622-627. https://doi. org/10.1002/acp.3239

Larkin, W. (1971). Response mechanisms in detection experiments. Journal of Experimental Psychology, 91(1), 140-153. https://doi.org/10.1037/h0031799

Megreya, A. M., \& Burton, A. M. (2007). Hits and false positives in face matching: A familiaritybased dissociation. Perception \& Psychophysics, 69, 1175-1184. https://doi.org/10.3758/ BF03193954

Palermo, R., Rossion, B., Rhodes, G., Laguesse, R., Hall, B., Albonico, A., . . Mckone, E. (2017). Do people have insight into their face recognition abilities? The Quarterly Journal of Experimental Psychology, 70, 218-233. https://doi.org/10.1080/17470218.2016.1161058

Ramon, M., Bobak, A., \& White, D. (2019). Super-recognizers: From the lab to the world and back again. British Journal of Psychology, 110, 461-497. https://doi.org/10.1111/bjop.12368

Reedy, M., \& Devue, C. (2019). Exposure to variations in appearance fast-tracks familiarisation with novel faces, but only in good recognisers. OSF, February 2. https://doi.org/10.31219/osf. io/6s8c7

Received 24 February 2019 